\title{
IMMUNOTHERAPY WITH INTERLEUKIN-2: A STUDY BASED ON MATHEMATICAL MODELING
}

\author{
SANDIP BANERJEE \\ Indian Institute of Technology and Science Rooorkee (IITR) \\ Roorkee 247667, Uttarakhand, India \\ e-mail: sandofma@iitr.ernet.in
}

\begin{abstract}
The role of interleukin-2 (IL-2) in tumor dynamics is illustrated through mathematical modeling, using delay differential equations with a discrete time delay (a modified version of the Kirshner-Panetta model). Theoretical analysis gives an expression for the discrete time delay and the length of the time delay to preserve stability. Numerical analysis shows that interleukin-2 alone can cause the tumor cell population to regress.
\end{abstract}

Keywords: effector cells, tumor cells, interleukin-2, discrete time delay.

\section{Introduction}

The mechanism of the establishment and destruction of cancer, one of the greatest killer of the world, is still a puzzle. Modern treatments involve surgery, chemotherapy and radiotherapy, but yet relapses occur. Hence, the need for a more successful treatment is very obvious. Developing schemes for immunotherapy or its combination with other therapy methods are the major attempts at present, which aim at reducing the tumor mass, heightening tumor immunogenicity and removing the immunosuppression induced in an organism in the process of tumor growth. Recent progress in this line has been achieved through immunotherapy, which refers to the use of cytokines (protein hormones that mediate both natural and specific immunity) usually together with adoptive cellular immunotherapy (ACI) (Rosenberg and Lotze, 1986; Schwartzentruber, 1993; Rosenberg et al., 1994; Keilholz et al., 1994; Gause et al., 1996; Hara et al., 1996; Kaempfer et al., 1996; Curti et al., 1996; Rabinowich et al., 1996).

The main cytokine responsible for lymphocyte activation, growth and differentiation is interleukin-2 (IL2), which is mainly produced by T-helper cells (CD4+ $\mathrm{T}$-cells) and in relatively small quantities by cytotoxic $\mathrm{T}$ lymphocytes (CD8+ T-cells). CD4 lymphocytes differentiate into T-Helper 1 and T-Helper 2 functional subjects due to the immune response. IL-2 acts in an autocrine manner on T-Helper 1 and also induces the growth of THelper 2 and CD8 lymphocytes in a paracrine manner.
The T-lymphocytes themselves are stimulated by the tumor to induce further growth. Thus, the complete biological assumption of adoptive cellular immunotherapy is that the immune system is expanded in number artificially (ex vivo) in cell cultures by means of human recombinant interleukin-2. This can be done in two ways, either by (i) a lymphokine-activated killer cell therapy (LAKtherapy), where the cells are obtained from in vitro culturing of peripheral blood leukocytes removed from patients with high concentration of IL-2, or (ii) a tumor infiltrating lymphocyte therapy (TIL), where the cells are obtained from lymphocytes recovered from the patient tumors, which are then incubated with high concentrations of IL-2 in vitro and are comprised of activated natural killer (NK) cells and cytotoxic T-lymphocyte CTL cells. The tumor infiltrating lymphocytes (TIL) are then put back into the bloodstream, along with IL-2, where they can bind to and destroy the tumor cells. It has been established clinically that immunotherapy with IL-2 has enhanced the cytotoxic T-lymphocyte (CTL) activity at different stages of tumor (Rosenberg and Lotze, 1986; Schwartzentruber, 1993; Rosenberg et al., 1994; Keilholz et al., 1994). Also, there is evidence of the restoration of the defective natural killer (NK) cell activity as well as the enhancement of polyclonal expansion of CD4+ and CD8+ T-cells (Rosenstein et al., 1986; Tartour et al., 1996). Figure 1 gives a schematic diagram showing the key players in tumorimmune interactions (http://www.rose-hulman.edu/mathjournal /archives/2005/vol6-n2/paper9/v6n2-9pd.pdf). 
Interaction between tumor cells and an immune system has been studied by numerous authors (Tartour et al., 1996; Kuznetsov et al., 1994; Adam and N. Bellomo, 1997; Kirschner and Panetta, 1998; Bodnar and Foryś, 2000b; Foryś, 2002; Galach, 2003; Kolev, 2003; Zhivkovc and Waniewski, 2003; Szymańska, 2003; Matzavinos et al., 2004; Sarkar and Banerjee, 2005; Banerjee and Sarkar, 2008). Kuznetsov et al. (1994) present a mathematical model of the cytotoxic T-lymphocyte response to the growth of an immunogenic tumor. The model exhibits a number of phenomena that are seen in vivo, including the immunostimulation of the tumor growth, "sneaking through" the tumor, and the formation of a tumor "dormant state". The delay version of Kuznetsov's model was studied by Galach (2003), where the effect of the time delay was taken into account in order to achieve a better compatibility with reality. In (Foryś, 2002), Marchuk's model of a general immune reaction is presented. Qualitative behavior of solutions to the model (and its simplification), along with many illustrations of the recovery process, oscillations or lethal outcomes of a disease, is shown. The role of interleukins in the immune process is taken into account to adapt Marchuk's model to tumor growth dynamics. Szymańska (2003) studied a basic mathematical model of the immune response when cancer cells are recognized. The model consists of six ordinary differential equations and is extended by taking into account two types of immunotherapy: an active immunotherapy and an adoptive immunotherapy. An analysis of the corresponding models is made to answer the question of which of the presented methods of immunotherapy is better. The analysis is completed by numerical simulations which show that the method of adoptive immunotherapy seems better for the patient at least in some cases. A mathematical model describing the growth of a solid tumor in the presence of an immune system response is presented by (Matzavinos et al., 2004). They focused upon the attack of tumor cells by the so-called tumor-infiltrating cytotoxic lymphocytes (TICLs), in a small, multicellular tumor, without necrosis and at some stage prior to (tumor induced) angiogenesis. Their study can explain the complex heterogeneous spatio-temporal dynamics observed and lead to a deeper understanding of the phenomenon of cancer dormancy in the model, which may be helpful in future development of more effective anti-cancer vaccines.

In (Sarkar and Banerjee, 2005), the authors express the spontaneous regression and progression of a malignant tumor system as a prey-predator-like system. Their model is a three dimensional deterministic system, consisting of tumor cells, hunting predator cells and resting predator cells, which is extended to a stochastic one, allowing for random fluctuations around the positive interior equilibrium. The stochastic stability properties of the model are investigated both analytically and numerically, and the thresholds obtained from their study may be helpful to control the malignant tumor growth. They also studied the model by including a discrete time delay in the system (Banerjee and Sarkar, 2008) and concluded that the model can provide an approximate estimate of timing (length of delay) and a dosage of therapy that would best complement the patient's own defense mechanism versus the tumor cells. More work on time delays in connection with tumor growth can be found in (Byrne, 1997; Bodnar and Foryś, 2000a; Bodnar and Foryś, 2003a; Bodnar and Foryś, 2003b).

Kirschner and Penetta studied the role of IL-2 in tumor dynamics, particularly, long-term tumor recurrence and short term oscillations in a mathematical perspective (Kirschner and Panetta, 1998). The model proposed there deals with three populations, namely, the activated immune-system cells (commonly called the effector cells), such as cytotoxic T-cells, macrophages and natural killer cells that are cytotoxic to the tumor cells, the tumor cells and the concentration of IL-2. The important parameters in their study are the antigenicity of tumor $(c)$, the treatment term that represents the external source of effector cells $\left(s_{1}\right)$ and the treatment term that represents an external input of IL-2 into the system $\left(s_{2}\right)$. Their results can be summarized as follows: (i) For a non-treatment case $\left(s_{1}=0, s_{2}=0\right)$, the immune system is not able to clear the tumor for low antigenic tumors while for highly antigenic tumors, reduction to a small dormant tumor is the best case scenario. (ii) The effect of adoptive cellular immunotherapy (ACI) therapy $\left(s_{1}>0, s_{2}=0\right)$ alone can yield a tumor free state for tumors of almost any antigenicity, provided the treatment concentration is above a given critical level. But for tumors with small antigenicity, an early treatment is needed, while the tumor is small, so that the tumor can be controlled. (iii) The treatment with IL-2 alone $\left(s_{1}=0, s_{2}>0\right)$ states that if IL-2 administration is low, there is no tumor-free state. However, if the IL-2 input is high, the tumor can be cleared but the immune system grows without bounds causing problems such as a capillary leak syndrome. (iv) Finally, it is the combined treatment with ACI and IL-2 $\left(s_{1}>0, s_{2}>0\right)$ that gives the combined effects obtained from the monotherapy regime. For any antigenicity, there is a region of tumor clearance. These results indicate that a treatment with ACI may be a better option either as a monotherapy or in conjunction with IL-2.

In this paper, a modification of the model studied by Kirschner and Panetta is done, by adding a discrete time delay which exists when activated T-cells produce IL- 2 . The modified model is discussed in Section 2. Section 3 deals with the qualitative analysis of the model. In Section 4, the numerical results are discussed, and Section 5 is the conclusion. 


\section{Model}

The idea of the model presented in this paper came from the paper by Kirschner and Panetta (1998), where the equations representing the model are

$$
\begin{aligned}
\frac{\mathrm{d} E}{\mathrm{~d} t_{1}} & =c T+\frac{p_{1} E I_{L}}{g_{1}+I_{L}}-\mu_{2} E+s_{1}, \\
\frac{\mathrm{d} T}{\mathrm{~d} t_{1}} & =r_{2}(1-b T) T-\frac{a E T}{g_{2}+T}, \\
\frac{\mathrm{d} I_{L}}{\mathrm{~d} t_{1}} & =\frac{p_{2} E T}{g_{3}+T}-\mu_{3} I_{L}+s_{2},
\end{aligned}
$$

with initial conditions $E(0)=E_{0}, T(0)=T_{0}, I_{L}(0)=$ $I_{L 0}$.

The model, which had been represented by ordinary differential equations in (Kirschner and Panetta, 1998), was extended to delay differential equations (in nondimensionalized form) with proper biological justifications. The equations representing the system are

$$
\begin{aligned}
& \frac{\mathrm{d} x}{\mathrm{~d} t}=c y+\frac{p_{1} x(t-\tau) z(t-\tau)}{g_{1}+z(t-\tau)}-\mu_{2} x+s_{1}, \\
& \frac{\mathrm{d} y}{\mathrm{~d} t}=r_{2}(1-b y) y-\frac{a x y}{g_{2}+y}, \\
& \frac{\mathrm{d} z}{\mathrm{~d} t}=\frac{p_{2} x y}{g_{3}+y}-\mu_{3} z+s_{2},
\end{aligned}
$$

subject to the following initial conditions:

$$
\begin{aligned}
x(\theta) & =\psi_{1}(\theta), \quad y(\theta)=\psi_{2}(\theta), \quad z(\theta)=\psi_{3}(\theta), \\
\psi_{1}(\theta) & \geq 0, \quad \psi_{2}(\theta) \geq 0, \quad \psi_{3}(\theta) \geq 0, \quad \theta \in[-\tau, 0], \\
\psi_{1}(0) & >0, \quad \psi_{2}(0)>0, \quad \psi_{3}(0)>0,
\end{aligned}
$$

where $C_{+}=\left(\psi_{1}(\theta), \psi_{2}(\theta), \psi_{3}(\theta)\right) \in C\left([-\tau, 0], \mathbb{R}_{+0}^{3}\right)$, the Banach space of continuous functions mapping the interval $[-\tau, 0]$ into $\mathbb{R}_{+0}^{3}$, where $\mathbb{R}_{+0}^{3}$ is defined as

$$
\mathbb{R}_{+0}^{3}=((x, y, z): x, y, z \geq 0)
$$

and $\mathbb{R}_{+}^{3}$, the interior of $\mathbb{R}_{+0}^{3}$, as

$$
\mathbb{R}_{+}^{3}=((x, y, z): x, y, z>0) .
$$

The system given by (10) is non-dimensionalized using the following scaling (Kirschner and Panetta, 1998):

$$
\begin{aligned}
& x=E / E_{0}, \quad y=T / T_{0}, \quad z=I_{L} / I_{L_{0}}, \\
& t=t_{s} t_{1}, \quad \bar{c}=c T_{0} / t_{s} E_{0}, \quad \overline{p_{1}}=p_{1} / t_{s}, \\
& \overline{g_{1}}=g_{1} / I_{L_{0}}, \quad \overline{\mu_{2}}=\mu_{2} / t_{s}, \quad \overline{g_{2}}=g_{2} / T_{0}, \\
& \bar{b}=b T_{0}, \quad \bar{a}=a E_{0} / t_{s} T_{0}, \quad \overline{r_{2}}=r_{2} / t_{s}, \\
& \overline{\mu_{3}}=\mu_{3} / t_{s}, \quad \overline{p_{2}}=p_{2} E_{0} / t_{s} I_{L_{0}}, \quad \overline{g_{3}}=g_{3} / T_{0} \text {, } \\
& \overline{s_{1}}=s_{1} / t_{s} E_{0}, \quad \overline{s_{2}}=s_{2} / t_{s} I_{L_{0}} \text {. }
\end{aligned}
$$

For convenience, the over-bar notation is dropped and the scaled model is given by (1). A proper scaling is needed as the system is numerically stiff and numerical routines used to solve these equations will fail without scaling or with inappropriate scaling. (In this case, a proper choice of scaling is $E_{0}=T_{0}=I_{L_{0}}=1 / b$ and $t_{s}=r_{2}$ (Kirschner and Panetta, 1998)). The parameter values were obtained from (Kirschner and Panetta, 1998), which is put in tabular form (Table 1). The units of the parameters are in day ${ }^{-1}$, except of $g_{1}, g_{2}, g_{3}$ and $b$, which are in volumes.

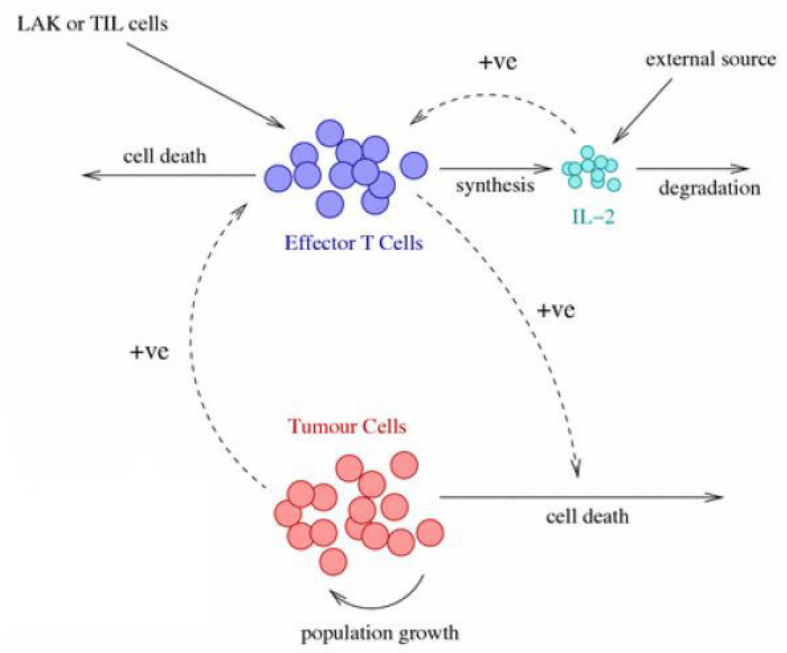

Fig. 1. Schematic diagram showing the key-players in tumor-immune interactions.

In the system described by (1), $x(t), y(t)$ and $z(t)$ respectively represent the effector cells, the tumor cells and the concentration of IL-2 in a single site compartment. The first equation of the system (1) describes the rate of change in the effector cell population. The effector cells grow due to the direct presence of the tumor, given by the term $c y$, where $c$ is the antigenicity of the tumor. It is also stimulated by IL-2 that is produced by effector cells in an autocrine and paracrine manner (the term $p_{1} x z /\left(g_{1}+\right.$ $z), p_{1}$ is the rate at which the effector cells grows and $g_{1}$ is the half saturation constant). A clinical trial shows that there are immune stimulation effects from treatment with IL-2 (Keilholz et al., 1994; Gause et al., 1996; Hara et al., 1996; Kaempfer et al., 1996; Curti et al., 1996), and there is a time lag between the production of interleukin-2 by activated T-cells and the effector cell stimulation from treatment with IL-2. Hence, a discrete time delay is being added to the second term of the first equation of the system (11), which modifies to $p_{1} x(t-\tau) z(t-\tau) /\left(g_{1}+z(t-\tau)\right)$, $\mu_{2} x$ gives the natural decay of the effector cells and $s_{1}$ is the treatment term that represents the external source of the effector cells such as adoptive cellular immunotherapy (ACI). A similar kind of term was introduced by Galach (2003) in his model equation, where he assumed that the source of the effector cells is the term $x(t-\tau) y(t-\tau)$, as 
Table 1. Parameter values used for numerical analysis.

\begin{tabular}{|c|c|c|}
\hline Parameters & Values & Scales Values \\
\hline \hline$c$ (antigenicity of tumor) & $0 \leq c \leq 0.05$ & $0 \leq c \leq 0.278$ \\
\hline$p_{1}$ (growth rate of effector cells) & 0.1245 & 0.69167 \\
\hline$g_{1}$ (half saturation constant) & $2 \times 10^{7}$ & 0.02 \\
\hline$\mu_{2}$ (natural decay rate of effector cells) & 0.03 & 0.1667 \\
\hline$r_{2}$ (growth rate of tumor cells) & 0.18 & 1 \\
\hline$b$ (1/carrying capacity of tumor cells) & $1.0 \times 10^{-9}$ & 1 \\
\hline$a$ (decay rate of tumor) & 1 & 5.5556 \\
\hline$g_{2}$ (half saturation constant) & $1 \times 10^{5}$ & 0.0001 \\
\hline$\mu_{3}$ (natural decay rate of IL-2) & 10 & 55.556 \\
\hline$p_{2}$ (growth rate of IL-2) & 5 & 27.778 \\
\hline$g_{3}$ (half saturation constant) & $1 \times 10^{3}$ & 0.000001 \\
\hline
\end{tabular}

the immune system needs some time to develop a suitable response.

The second equation of the system (1) shows the rate of change of the tumor cells which follows a logistic growth (a type of limiting growth). Due to a tumoreffector cell interaction, there is a loss in the tumor cells at the rate $a$ and it is modeled by Michaelis-Menten kinetics to indicate the limited immune response to the tumor (the term $\operatorname{axy} /\left(g_{2}+y\right), g_{2}$ being a half saturation constant). The third equation of the system (1) gives the rate of change for the concentration of IL-2. Its source are the effector cells, which are stimulated by interaction with the tumor and also have Michaelis-Menten kinetics to account for the self-limiting production of IL-2 (the term $p_{2} x y /\left(g_{3}+y\right), p_{2}$ being the rate of production of IL-2 and $g_{3}$ a half saturation constant), $\mu_{3} z$ is the natural decay of the IL-2 concentration and $s_{2}$ is a treatment term that represents an external input of IL-2 into the system.

The aim of this paper is to study this modified model and to explore any changes in the dynamics of the system that may occur when a discrete time delay is added to the system, and to compare the results with those obtained by Kirschner and Panetta (1998).

\section{Qualitative analysis of the model}

3.1. Positivity of the solution. The system of equations is now put in vector form by setting

$$
\begin{aligned}
X & =\operatorname{col}(M, N, Z) \in \mathbb{R}_{+0}^{3}, \\
F(X) & =\left(\begin{array}{l}
F_{1}(X) \\
F_{2}(X) \\
F_{3}(X)
\end{array}\right)
\end{aligned}
$$

$$
=\left(\begin{array}{c}
c y+\frac{p_{1} x(t-\tau) z(t-\tau)}{g_{1}+z(t-\tau)}-\mu_{2} x+s_{1} \\
r_{2}(1-b y) y-\frac{a x y}{g_{2}+y} \\
\frac{p_{2} x y}{g_{3}+y}-\mu_{3} z+s_{2}
\end{array}\right),
$$

where $F: C_{+} \rightarrow \mathbb{R}_{+0}^{3}$ and $F \in C^{\infty}\left(\mathbb{R}_{+0}^{3}\right)$. Then the system (1) becomes

$$
\dot{X}=F\left(X_{t}\right),
$$

where $. \equiv \mathrm{d} / \mathrm{d} t$ and with $X_{t}(\theta)=X(t+\theta), \theta \in[-\tau, 0]$ (Hale and Lunel, 1993). It is easy to check in (4) that, whenever we choose $X(\theta) \in C_{+}$such that $X_{i}=0$, then we obtain $\left.F_{i}(X)\right|_{X_{i}(t)=0, X_{t} \in C_{+}} \geq 0, i=1,2,3$. Due to the lemma in (Yang et al., 1996), any solution of (4) with $X(\theta) \in C_{+}$, say, $X(t)=X(t, X(0))$, is such that $X(t) \in \mathbb{R}_{+0}^{3}$ for all $t>0$.

3.2. Linear stability analysis with delay. The equilibria for the system (scaled) are as follows:

(i) The $x-z$ planar equilibrium is

$$
\left(\frac{s_{1}\left(g_{1} \mu_{3}+s_{2}\right)}{\mu_{2}\left(g_{1} \mu_{3}+s_{2}\right)-p_{1} s_{2}}, 0, \frac{s_{2}}{\mu_{3}}\right)
$$

and exists if

$$
\mu_{2}>\frac{p_{1} s_{2}}{g_{1} \mu_{3}+s_{2}} .
$$

(ii) The interior equilibrium is $E_{*}\left(x^{*}, y^{*}, z^{*}\right)$, where

$$
\begin{gathered}
x^{*}=\frac{r_{2}}{a}\left(1-b y^{*}\right)\left(g_{2}+y^{*}\right), \\
z^{*}=\frac{p_{2} r_{2}\left(1-b y^{*}\right)\left(g_{2}+y^{*}\right)}{a \mu_{3}\left(g_{3}+y^{*}\right)}+\frac{s_{2}}{\mu_{3}}
\end{gathered}
$$


and $y^{*}$ is given by

$$
c y^{*}-\mu_{2} x^{*}+\frac{p_{1} x^{*} z^{*}}{g_{1}+z^{*}}+s_{1}=0 .
$$

In the case of a positive delay, the characteristic equation for the linearized equation around the point $\left(x^{*}, y^{*}, z^{*}\right)$ is

$$
P(\lambda)+Q(\lambda) e^{-\lambda \tau}=0
$$

where

$$
\begin{aligned}
& P(\lambda)=\lambda^{3}+a_{1} \lambda^{2}+a_{2} \lambda+a_{3} \\
& Q(\lambda)=b_{1} \lambda^{2}+b_{2} \lambda+b_{3} \\
& a_{1}=\mu_{2}+\mu_{3}+b r_{2} y^{*}-\frac{a x^{*} y^{*}}{\left(g_{2}+y^{*}\right)^{2}} \\
& a_{2}=b r_{2} y^{*}\left(\mu_{3}-\frac{p_{1} z^{*}}{g_{1}+z^{*}}\right)+\frac{a p_{1} x^{*} y^{*} z^{*}}{\left(g_{2}+y^{*}\right)^{2}\left(g_{1}+z^{*}\right)} \\
& -\frac{\mu_{3} p_{1} z^{*}}{g_{1}+z^{*}}+\frac{a c y^{*}}{\left(g_{2}+y^{*}\right)} \\
& +\mu_{2}\left\{\mu_{3}+b r_{2} y^{*}-\frac{a x^{*} y^{*}}{\left(g_{2}+y^{*}\right)^{2}}\right\} \\
& a_{3}=b \mu_{2} \mu_{3} r_{2} y^{*}-\frac{a \mu_{2} \mu_{3} x^{*} y^{*}}{\left(g_{2}+y *\right)^{2}} \\
& +\frac{a c \mu_{3} y^{*}}{\left(g_{2}+y^{*}\right)} \\
& +\frac{a g_{1} g_{3} p_{1} p_{2}\left(x^{*}\right)^{2} y^{*}}{\left(g_{2}+y^{*}\right)\left(g_{3}+y^{*}\right)^{2}\left(g_{1}+z^{*}\right)^{2}} \\
& -\frac{b g_{1} p_{1} p_{2} r_{2} x^{*}\left(y^{*}\right)^{2}}{\left(g_{3}+y^{*}\right)\left(g_{1}+z^{*}\right)^{2}} \\
& +\frac{a g_{1} p_{1} p_{2}\left(x^{*}\right)^{2}\left(y^{*}\right)^{2}}{\left(g_{2}+y^{*}\right)^{2}\left(g_{3}+y^{*}\right)\left(g_{1}+z^{*}\right)^{2}} \\
& -\frac{b \mu_{3} p_{1} r_{2} y^{*} z^{*}}{g_{1}+z^{*}}+\frac{a \mu_{3} p_{1} x^{*} y^{*} z^{*}}{\left(g_{2}+y^{*}\right)^{2}\left(g_{1}+z^{*}\right)}, \\
& b_{1}=-\frac{p_{1} z^{*}}{g_{1}+z^{*}}, \\
& b_{2}=-\frac{g_{1} p_{1} x^{*} y^{*}}{\left(g_{3}+y^{*}\right)\left(g_{1}+z^{*}\right)^{2}}<0, \\
& b_{3}=\frac{a g_{1} p_{1} p_{2}\left\{g_{2} g_{3}+2 g_{3} y^{*}+(y *)^{2}\right\} x^{*} y^{*}}{\left(g_{2}+y^{*}\right)^{2}\left(g_{3}+y^{*}\right)^{2}\left(g_{1}+z^{*}\right)^{2}} \\
& -\frac{b r_{2} g_{1} p_{1} p_{2} x^{*}\left(y^{*}\right)^{2}}{\left(g_{3}+y^{*}\right)\left(g_{1}+z^{*}\right)^{2}} \text {. }
\end{aligned}
$$

The steady state is stable in the absence of the delay $(\tau=$ 0 ) if the roots of

$$
\begin{array}{r}
P(\lambda)+Q(\lambda)=0 \\
\Rightarrow \lambda^{3}+\left(a_{1}+b_{1}\right) \lambda^{2}+\left(a_{2}+b_{2}\right) \lambda+a_{3}+b_{3}=0
\end{array}
$$

have negative real parts. This occurs if and only if $a_{1}+$ $b_{1}>0, a_{3}+b_{3}>0$ and $\left(a_{1}+b_{1}\right)\left(a_{2}+b_{2}\right)-\left(a_{3}+b_{3}\right)>0$ (by Routh Hurwitz's criteria). This implies

$$
\begin{aligned}
& \mu_{2}+\mu_{3}+b r_{2} y^{*}-\frac{a x^{*} y^{*}}{\left(g_{2}+y *\right)^{2}}-\frac{p_{1} z^{*}}{g_{1}+z^{*}}>0, \\
& p_{1}\left\{\frac{g_{1} p_{2} x^{*} y^{*}}{\mu_{3}\left(g_{3}+y^{*}\right)\left(g_{1}+z^{*}\right)^{2}}+\frac{z^{*}}{g_{1}+z^{*}}\right\}<\mu_{2} \\
& <p_{1}\left\{\frac{g_{1} p_{2} x^{*}\left(g_{2} g_{3}+2 g_{3} y^{*}+(y *)^{2}\right)}{\mu_{3}\left(g_{3}+y^{*}\right)^{2}\left(g_{1}+z^{*}\right)^{2}}+\frac{z^{*}}{g_{1}+z^{*}}\right\} .
\end{aligned}
$$

(The above criteria are satisfied with the set of parameters shown in Table 1, provided that $0 \leq c \leq 0.278, s_{2}<$ $\mu_{2} \mu_{3} g_{1} /\left(p_{1}-\mu_{2}\right)$ ). Now substituting $\lambda=i \omega$ (where $\omega$ is positive) in Eqn. (5) and separating the real and imaginary parts, we obtain the system of transcendental equations

$$
\begin{aligned}
a_{1} \omega^{2}-a_{3} & =\left(b_{3}-b_{1} \omega^{2}\right) \cos (\omega \tau)+b_{2} \omega \sin (\omega \tau),(7) \\
\omega^{3}-a_{2} \omega & =b_{2} \omega \cos (\omega \tau)-\left(b_{3}-b_{1} \omega^{2}\right) \sin (\omega \tau)
\end{aligned}
$$

Squaring and adding (7) and (8), we get

$$
\begin{array}{r}
\left(b_{3}-b_{1} \omega^{2}\right)^{2}+b_{2}^{2} \omega^{2}=\left(a_{1} \omega^{2}-a_{3}\right)^{2}+\left(\omega^{3}-a_{2} \omega\right)^{2} \\
\Rightarrow \rho^{3}+A_{1} \rho^{2}+A_{2} \rho+A_{3}=0,
\end{array}
$$

where $\rho=\omega^{2}$,

$$
\begin{aligned}
& A_{1}=a_{1}^{2}-2 a_{2}-b_{1}^{2} \\
& =\mu_{2}^{2}+\mu_{3}^{2}+b^{2} r_{2}^{2}\left(y^{*}\right)^{2}+\frac{a^{2}\left(x^{*}\right)^{2}\left(y^{*}\right)^{2}}{\left(g_{2}+y^{*}\right)^{4}} \\
& -\frac{2 a y^{*}\left\{b r_{2} x^{*} y^{*}+c\left(g_{2}+y^{*}\right)\right\}}{\left(g_{2}+y^{*}\right)^{2}}+\frac{p_{1}^{2}\left(z^{*}\right)^{2}}{\left(g_{1}+z^{*}\right)^{2}} \\
& -\frac{2 \mu_{2} p_{1} z^{*}}{g_{1}+z^{*}} \\
& A_{2}=a_{2}^{2}-b_{2}^{2}-2 a_{1} a_{3}+2 b_{1} b_{3} \\
& =\frac{-g_{1}^{2} p_{1}^{2} p_{2}^{2}\left(x^{*}\right)^{2}\left(y^{*}\right)^{2}}{\left(g_{3}+y^{*}\right)^{2}\left(g_{1}+z^{*}\right)^{4}}+\left\{\frac{-a \mu_{3} x^{*} y^{*}}{\left(g_{2}+y *\right)^{2}}+\frac{a c y^{*}}{g_{2}+y *}\right. \\
& +\mu_{2}\left(\mu_{3}+b r_{2} y^{*}-\frac{-a x^{*} y^{*}}{\left(g_{2}+y *\right)^{2}}\right)-\frac{\mu_{3} p_{1} z^{*}}{g_{1}+z *} \\
& \left.+\frac{a p_{1} x^{*} y^{*} z^{*}}{\left(g_{2}+y^{*}\right)^{2}\left(g_{1}+z^{*}\right)}+b r_{2} y^{*}\left(\mu_{3}-\frac{p_{1} z^{*}}{g_{1}+z}\right)\right\}^{2} \\
& -2 \mu_{3} y^{*}\left[\mu_{2}+\mu_{3}+b r_{2} y^{*}-\frac{a x^{*} y^{*}}{\left(g_{2}+y *\right)^{2}}-\frac{p_{1} z^{*}}{g_{1}+z^{*}}\right] \\
& \times\left[b r _ { 2 } \left(\mu_{2}-\frac{p_{1} z^{*}}{g_{1}+z^{*}}\right.\right. \\
& \left.\left.+\frac{a\left\{-\mu_{2} x+c\left(g_{2}+y\right)+\frac{\mu_{3} p_{1} z^{*}}{g_{1}+z *}\right\}}{\left(g_{2}+y^{*}\right)^{2}}\right)\right] \text {, }
\end{aligned}
$$




$$
\begin{aligned}
A_{3}= & a_{3}^{2}-b_{3}^{2}=\left(a_{3}+b_{3}\right)\left(a_{3}-b_{3}\right) \\
= & y^{*}\left[b r_{2}\left\{\mu_{2} \mu_{3}-\frac{g_{1} p_{1} p_{2} x^{*} y^{*}}{\left(g_{3}+y *\right)\left(g_{1}+z^{*}\right)^{2}}-\frac{\mu_{3} p_{1} z^{*}}{g_{1}+z^{*}}\right\}\right. \\
& +\frac{a}{\left(g_{2}+y^{*}\right)^{2}}\left\{c \mu_{3}\left(g_{2}+y^{*}\right)\right. \\
& +x^{*}\left(-\mu_{2} \mu_{3}+\frac{p_{1} p_{2} g_{1} x^{*}\left(g_{2} g_{3}+2 g_{3} y^{*}+\left(y^{*}\right)^{2}\right)}{\left(g_{3}+y^{*}\right)^{2}\left(g_{1}+z *\right)^{2}}\right. \\
& \left.\left.+\frac{\mu_{3} p_{1} z^{*}}{g_{1}+z^{*}}\right\}\right] y^{*}\left[b r _ { 2 } \left\{\mu_{2} \mu_{3}\right.\right. \\
& \left.+\frac{g_{1} p_{1} p_{2} x^{*} y^{*}}{\left(g_{3}+y^{*}\right)\left(g_{1}+z^{*}\right)^{2}}-\frac{\mu_{3} p_{1} z^{*}}{g_{1}+z^{*}}\right\} \\
& +\frac{a}{\left(g_{2}+y^{*}\right)^{2}}\left\{c \mu_{3}\left(g_{2}+y^{*}\right)+x^{*}\left(-\mu_{2} \mu_{3}\right.\right. \\
& +\frac{-p_{1} p_{2} g_{1} x^{*}\left(g_{2} g_{3}+2 g_{3} y^{*}+\left(y^{*}\right)^{2}\right)}{\left(g_{3}+y *\right)^{2}\left(g_{1}+z *\right)^{2}} \\
& \left.\left.+\frac{\mu_{3} p_{1} z^{*}}{g_{1}+z^{*}}\right\}\right] .
\end{aligned}
$$

Assuming that $A_{1}$ is positive (this is satisfied with the parameter values from Table 1), the simplest assumption that (9) will have a positive root is $A_{3}=a_{3}^{2}-b_{3}^{2}<0$. Since $a_{3}+b_{3}$ is positive (from the non-delay case), we must have $a_{3}-b_{3}<0$ and this gives

$$
\begin{gathered}
y^{*}\left[b r_{2}\left\{\mu_{2} \mu_{3}+\frac{p_{1}\left(\frac{g_{1} p_{2} x^{*} y^{*}}{g_{3}+y^{*}}-\mu_{3} z^{*}\left(g_{1}+z^{*}\right)\right)}{\left(g_{1}+z^{*}\right)^{2}}\right\} b r_{2} y^{*}\right. \\
\left.-\frac{a x^{*} y^{*}}{\left(g_{2}+y *\right)^{2}}-\frac{p_{1} z^{*}}{g_{1}+z^{*}}\right]>0, \\
p_{1}\left\{\frac{g_{1} p_{2} x^{*} y^{*}}{\mu_{3}\left(g_{3}+y^{*}\right)\left(g_{1}+z^{*}\right)^{2}}+\frac{z^{*}}{g_{1}+z^{*}}\right\} \\
<\mu_{2}<p_{1}\left\{\frac{g_{1} p_{2} x^{*}\left(g_{2} g_{3}+2 g_{3} y^{*}+(y *)^{2}\right)}{\mu_{3}\left(g_{3}+y^{*}\right)^{2}\left(g_{1}+z^{*}\right)^{2}}\right. \\
\left.+\frac{z^{*}}{g_{1}+z^{*}}\right\} .
\end{gathered}
$$

Hence, we can say that there is a positive $\omega_{0}$ satisfying (9), that is, the characteristic equation (5) has a pair of purely imaginary roots of the form $\pm i \omega_{0}$. Eliminating $\sin (\tau \omega)$ from $(7)$ and $(8)$, we get

$$
\cos (\omega \tau)=\frac{\left(a_{1} \omega^{2}-a_{3}\right)\left(b_{3}\right)+\left(\omega^{3}-a_{2} \omega\right)\left(b_{2} \omega\right)}{\left(b_{3}\right)^{2}+\left(b_{2} \omega\right)^{2}} .
$$

Then $\tau_{n}^{*}$ corresponding to $\omega_{0}$ is given by

$$
\begin{aligned}
& \tau_{n}^{*} \\
& =\frac{1}{\omega_{0}} \arccos \left[\frac{\left(a_{1} \omega_{0}^{2}-a_{3}\right)\left(b_{3}\right)+\left(\omega_{0}^{3}-a_{2} \omega_{0}\right)\left(b_{2} \omega_{0}\right)}{\left(b_{3}\right)^{2}+\left(b_{2} \omega_{0}\right)^{2}}\right] \\
& \quad+\frac{2 n \pi}{\omega_{0}} .
\end{aligned}
$$

For $\tau=0, E_{*}$ is stable. Hence, $E_{*}$ will remain stable for $\tau<\tau_{0}$ where $\tau_{0}=\tau_{0}^{*}$ as $n=0$ (Freedman and Rao, 1983).

3.3. Estimation of the length of delay to preserve stability. The linearized form of the system (11) is

$$
\begin{aligned}
\frac{\mathrm{d} x}{\mathrm{~d} t} & =\left(\frac{p_{1} z^{*}}{g_{1}+z^{*}}-\mu_{2}\right) x+\frac{p_{1} z^{*}}{g_{1}+z^{*}} x(t-\tau) \\
& +c y+\frac{p_{1} g_{1} x^{*}}{\left(g_{1}+z^{*}\right)^{2}} z(t-\tau), \\
\frac{\mathrm{d} y}{\mathrm{~d} t} & =-\frac{a y^{*}}{g_{2}+y^{*}} x+\left(\frac{a x^{*} y^{*}}{\left(g_{2}+y^{*}\right)^{2}}-r_{2} b y^{*}\right) y, \\
\frac{\mathrm{d} z}{\mathrm{~d} t} & =-\frac{p_{2} y^{*}}{g_{3}+y^{*}} x+\frac{p_{2} g_{3} x^{*}}{\left(g_{3}+y^{*}\right)^{2}} y-\mu_{3} z .
\end{aligned}
$$

Taking the Laplace transform of the above linearized system, we get

$$
\begin{aligned}
& \left(s+\mu_{2}-\frac{p_{1} z^{*}}{g_{1}+z^{*}}\right) \bar{x}(s) \\
& =\frac{p_{1} z^{*}}{g_{1}+z^{*}} e^{-s \tau} \bar{x}(s)+\frac{p_{1} z^{*}}{g_{1}+z^{*}} e^{-s \tau} K_{1}(s) \\
& \quad+c \bar{y}(s)+\frac{p_{1} g_{1} x^{*}}{\left(g_{1}+z^{*}\right)^{2}} e^{-s \tau} \bar{z}(s) \\
& \quad+\frac{p_{1} g_{1} x^{*}}{\left(g_{1}+z^{*}\right)^{2}} e^{-s \tau} K_{2}(s)+x(0), \\
& \left(s+r_{2} b y^{*}-\frac{a x^{*} y^{*}}{\left(g_{2}+y^{*}\right)^{2}}\right) \bar{y}(s)=-\frac{a y^{*}}{g_{2}+y^{*}} \bar{x}(s)+y(0) \\
& \left(s+\mu_{3} z\right)=-\frac{p_{2} y^{*}}{g_{3}+y^{*}} \bar{x}(s)+\frac{p_{2} g_{3} x^{*}}{\left(g_{3}+y^{*}\right)^{2}} \bar{y}(s)+z(0)
\end{aligned}
$$

where

$$
\begin{aligned}
K_{1}(s) & =\int_{-\tau}^{0} e^{-s t} x(t) \mathrm{d} t, \\
K_{2}(s) & =\int_{-\tau}^{0} e^{-s t} z(t) \mathrm{d} t,
\end{aligned}
$$

and $\bar{x}(s), \bar{y}(s)$ and $\bar{z}(s)$ are the Laplace transforms of $x(t), y(t)$ and $z(t)$, respectively.

Following the lines of (Freedman et al., 1986) and using the Nyquist criterion (see the Appendix), it can be 

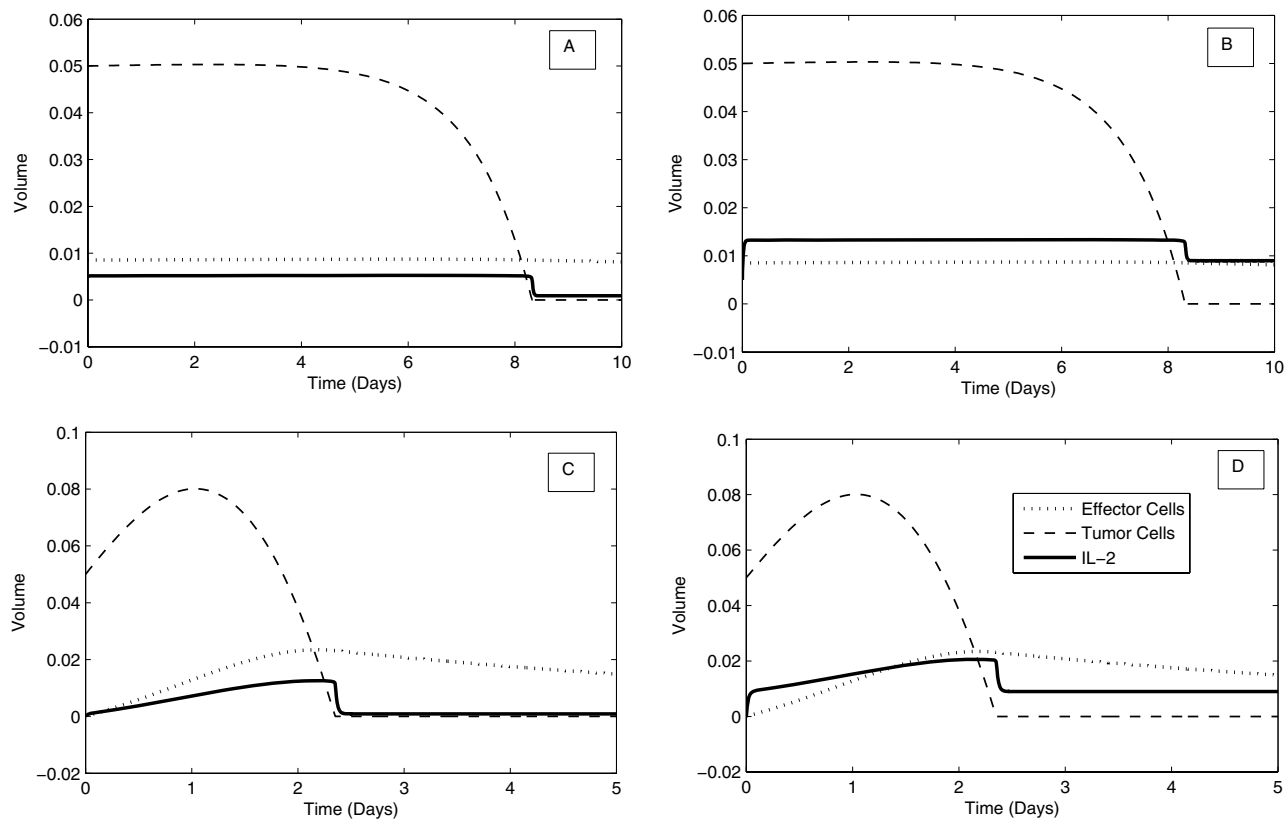

Fig. 2. Effector cells, tumor cells and IL-2 vs. time. All the parameter values were scaled accordingly. (a) $c=0.0056, s_{1}=0$, $s_{2}=0.05$; (b): $c=0.0056, s_{1}=0, s_{2}=0.2 ;$ (c): $c=0.222, s_{1}=0, s_{2}=0.05 ;$ (d): $c=0.222, s_{1}=0, s_{2}=0.2$; $\tau=\tau_{0}^{*}=0.723$ days $=17.346$ hours for Cases (a) and (b); $\tau=\tau_{0}^{*}=0.529$ days $=12.7$ hours for Cases (c) and (d).

shown that the conditions for the local asymptotic stability of $E_{*}\left(x^{*}, y^{*}, z^{*}\right)$ are given by

$$
\begin{aligned}
& \operatorname{Im} H\left(i \eta_{0}\right)>0, \\
& \operatorname{Re} H\left(i \eta_{0}\right)=0,
\end{aligned}
$$

where $H(s)=s^{3}+a_{1} s^{2}+a_{2} s+a_{3}+e^{-s \tau}\left(b_{1} s^{2}+b_{2} s+b_{3}\right)$ and $\eta_{0}$ is the smallest positive root of (12). In this case, (11) and (12) give

$$
\begin{aligned}
a_{2} \eta_{0}-\eta_{0}^{3}> & -b_{2} \eta_{0} \cos \left(\eta_{0} \tau\right)+b_{3} \sin \left(\eta_{0} \tau\right) \\
& -b_{1} \eta_{0}^{2} \sin \left(\eta_{0} \tau\right), \\
a_{3}-a_{1} \eta_{0}^{2}= & b_{1} \eta_{0}^{2} \cos \left(\eta_{0} \tau\right)-b_{3} \cos \left(\eta_{0} \tau\right) \\
& -b_{2} \eta_{0} \sin \left(\eta_{0} \tau\right) .
\end{aligned}
$$

Now, if Eqns. (13) and (14) are satisfied simultaneously, they are sufficient conditions to guarantee stability, which are now used to get an estimate to the length of the time delay. The aim is to find an upper bound $\eta_{+}$to $\eta_{0}$, independent of $\tau$, and then to estimate $\tau$ so that (113) holds true for all values of $\eta, 0 \leq \eta \leq \eta_{+}$and hence, in particular, at $\eta=\eta_{0}$.

Equation (14) is rewritten as

$$
\begin{aligned}
a_{1} \eta_{0}^{2}= & a_{3}+b_{3} \cos \left(\eta_{0} \tau\right)-b_{1} \eta_{0}^{2} \cos \left(\eta_{0} \tau\right) \\
& +b_{2} \eta_{0} \sin \left(\eta_{0} \tau\right) .
\end{aligned}
$$

\section{Maximizing}

$$
a_{3}+b_{3} \cos \left(\eta_{0} \tau\right)-b_{1} \eta_{0}^{2} \cos \left(\eta_{0} \tau\right)+b_{2} \eta_{0} \sin \left(\eta_{0} \tau\right),
$$

subject to

$$
\left|\sin \left(\eta_{0} \tau\right)\right| \leq 1, \quad\left|\cos \left(\eta_{0} \tau\right)\right| \leq 1,
$$

we obtain

$$
\left|a_{1}\right| \eta_{0}^{2} \leq\left|a_{3}\right|+\left|b_{3}\right|+\left|b_{1}\right| \eta_{0}^{2}+\left|b_{2}\right| \eta_{0} .
$$

Hence, if

$$
\begin{aligned}
\eta_{+}= & \frac{1}{2\left(\left|a_{1}\right|-\left|b_{1}\right|\right)}\left[\left|b_{2}\right|\right. \\
& \left.+\sqrt{b_{2}^{2}+4\left(\left|a_{1}\right|-\left|b_{1}\right|\right)\left(\left|a_{3}\right|+\left|b_{3}\right|\right)}\right],
\end{aligned}
$$

then clearly from (16) we have $\eta_{0} \leq \eta_{+}$.

From (13) we obtain

$$
\begin{aligned}
\eta_{0}^{2}< & a_{2}+b_{2} \cos \left(\eta_{0} \tau\right)+b_{1} \eta_{0} \sin \left(\eta_{0} \tau\right) \\
& -\frac{b_{3} \sin \left(\eta_{0} \tau\right)}{\eta_{0}} .
\end{aligned}
$$

Since $E_{*}\left(x^{*}, y^{*}, z^{*}\right)$ is locally asymptotically stable for $\tau=0$, for sufficiently small $\tau>0$, the inequality (18) will continue to hold. Substituting (15) in (18) and rearranging the result we get

$$
\begin{aligned}
\left(b_{3}-b_{1} \eta_{0}^{2}-a_{1} b_{2}\right)\left[\cos \left(\eta_{0} \tau\right)-1\right] & \\
+ & \left\{\left(b_{2}-a_{1} b_{1}\right) \eta_{0}+\frac{a_{1} b_{3}}{\eta_{0}}\right\} \sin \left(\eta_{0} \tau\right) \\
& <a_{1} a_{2}-a_{3}-b_{3}+b_{1} \eta_{0}^{2}+a_{1} b_{2} .
\end{aligned}
$$



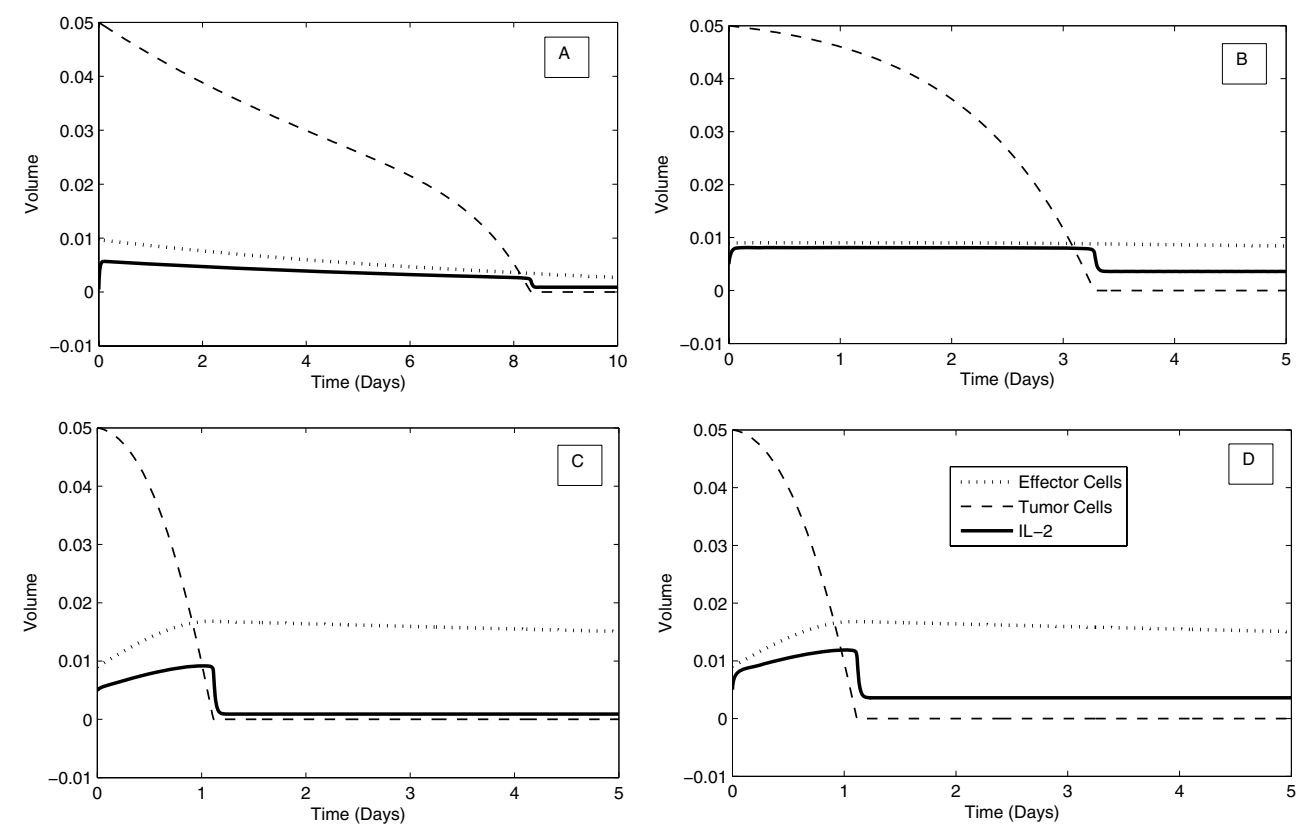

Fig. 3. Effector cells, tumor cells and IL-2 vs. time. All the parameter values were scaled accordingly. (a): $c=0.0056, s_{1}=$ $0.00000246446, s_{2}=0.05 ;$ (b): $c=0.0056, s_{1}=0.0000010144, s_{2}=0.2 ;$ (c): $c=0.222, s_{1}=0.00000246446$, $s_{2}=0.05 ;(\mathrm{d}): c=0.222, s_{1}=0.0000010144, s_{2}=0.2 ; \tau=\tau_{0}^{*}=0.7228$ days $=17.348$ hours for Cases (a) and (b); $\tau=\tau_{0}^{*}=0.528$ days $=12.67$ hours for Cases (c) and (d).

Using the bounds

$$
\begin{aligned}
&\left(b_{3}-b_{1} \eta_{0}^{2}-a_{1} b_{2}\right)[\left.\cos \left(\eta_{0} \tau\right)-1\right] \\
&=\left(b_{1} \eta_{0}^{2}+\right.\left.a_{1} b_{2}-b_{3}\right) 2 \sin ^{2}\left(\frac{\eta_{0} \tau}{2}\right) \\
& \leq \frac{1}{2}\left|\left(b_{1} \eta_{+}^{2}+a_{1} b_{2}-b_{3}\right)\right| \eta_{+}^{2} \tau^{2}
\end{aligned}
$$

and

$$
\begin{aligned}
\left\{\left(b_{2}-a_{1} b_{1}\right) \eta_{0}\right. & \left.+\frac{a_{1} b_{3}}{\eta_{0}}\right\} \sin \left(\eta_{0} \tau\right) \\
& \leq\left\{\left|\left(b_{2}-a_{1} b_{1}\right)\right| \eta_{+}^{2}+\left|a_{1}\right|\left|b_{3}\right|\right\} \tau
\end{aligned}
$$

from (19) we obtain

$$
L_{1} \tau^{2}+L_{2} \tau<L_{3}
$$

where

$$
\begin{aligned}
& L_{1}=\frac{1}{2}\left|\left(b_{1} \eta_{+}^{2}+a_{1} b_{2}-b_{3}\right)\right| \eta_{+}^{2}, \\
& L_{2}=\left|\left(b_{2}-a_{1} b_{1}\right)\right| \eta_{+}^{2}+\left|a_{1}\right|\left|b_{3}\right|, \\
& L_{3}=a_{1} a_{2}-a_{3}-b_{3}+b_{1}^{2} \eta_{+}+a_{1} b_{2} .
\end{aligned}
$$

Hence, if

$$
\tau_{+}=\frac{1}{2 L_{1}}\left(-L_{2}+\sqrt{L_{2}^{2}+4 L_{1} L_{3}}\right),
$$

then for $0 \leq \tau<\tau_{+}$the Nyquist criterion holds true and $\tau_{+}$estimates the maximum length of the delay preserving the stability.

\section{Numerical results}

The model is now studied numerically to see the effect of the discrete time delay on the system. The scaled parameter values have been used for numerical calculations using Matlab.

Case $1\left(s_{1}>0, s_{2}=0\right)$ : In the model, the time delay has no qualitative effect on the adaptive cellular immunotherapy (ACI). Therefore, the results will be the same as those obtained in (Kirschner and Panetta, 1998). So is the case $s_{1}=0, s_{2}=0$. Hence, these two cases are not discussed thoroughly.

Case $2\left(s_{1}=0, s_{2}>0\right)$ : Figure 2 explores the input of the concentration of IL-2 into the system, if the input of the concentration of IL-2 is administered and the effector cells are stimulated after 0.7227 days $=17.346$ hours and 0.529 days $=12.7$ hours, respectively (obtained by using (10) and scaled parameter values). For a low antigenic tumor and a low input of the concentration of IL-2 ( $c=0.0056$, $\left.s_{2}=0.05\right)$, the tumor cell regresses and the concentration of IL-2 decreases alarmingly almost to zero (Fig. 2(a)). For a higher concentration of IL-2 ( $\left.c=0.0056, s_{2}=0.2\right)$, the same scenario happens, and it is only in this case that the concentration of IL-2 does not reduce to zero (Fig. 2(b)).

For tumors with a high antigenicity $(c=0.222$, $\left.s_{2}=0.05\right)$, the tumor volume increases at the beginning 
and when there is an input of IL-2 concentration after 12.7 hours, the tumor volume reduces and ultimately is cleared off (Fig. 2(c)). At the same time, the concentration of Il2 decreases alarmingly. But with a high input of IL-2 on the tumor with a high antigenicity $\left(c=0.222, s_{2}=0.2\right)$, the tumor regresses as well, and the immune system and the concentration of IL-2 stabilize (Fig. 2(d)). This is a new interesting positive result. According to (Kirschner and Panetta, 1998), large amounts of administrated IL2 together with any degree of antigenicity show that the tumor is cleared but the immune system grows unbounded as the IL-2 concentration reaches a steady-state value (Fig. 4). This uncontrolled growth of the immune system represents a situation that is detrimental to the host. However, in our case, due to the time delay effect, the situation is under control. The tumor is cleared off and the immune system also stabilizes.

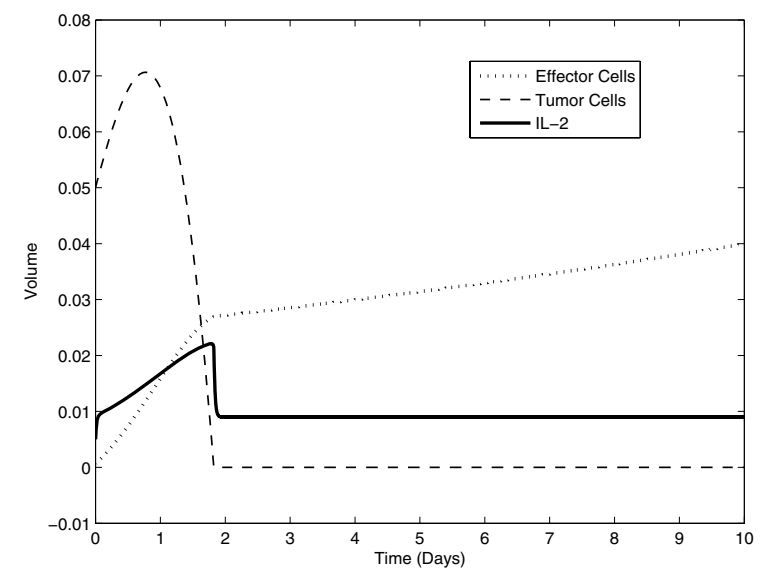

Fig. 4. Effector cells, tumor cells and IL-2 vs. time in the case of non-delay (i.e., $\tau=\tau_{0}^{*}=0$. All the parameter values were scaled accordingly. Here $c=0.222, s_{1}=0, s_{2}=0.5$.

Case $3\left(s_{1}>0, s_{2}>0\right)$ : Figure 3 shows the effect of immunotherapy with both ACI and IL-2, if the input of both ACI and the concentration of IL-2 are administered and the stimulation of the effector cells by IL-2 takes place after 0.7228 days $=17.348$ hours and 0.528 days $=12.67$ hours, respectively. Irrespective of the antigenicity of the tumor, the dynamics of Figs. 3(a)-3(d) are same, i.e., the volume of the tumor decreases significantly when both ACI and IL-2 are administered in various concentrations.

\section{Conclusion}

The aim of this paper was to see the effect of time delay during immunotherapy with interleukin-2 (IL-2). The effect of immunotherapy with IL-2 on the modified model was explored and circumstances under which the tumor can be eliminated are described. The model represented by a set of delay differential equations contains treatment terms $s_{1}$ and $s_{2}$ that represent an external source of the effector cells by adoptive cellular immunotherapy (ACI) and an external input of IL-2 into the system, respectively. However, the effects of IL-2 on the tumor-immune dynamics with time delay are the main focus. It is shown that treatment with IL-2 alone can offer a satisfactory outcome. When there is an external input of the concentration of IL2 and the effector cells are stimulated after 96.38 hours, during which the IL-2 production reaches its peak value to generate more effector cells, a tumor with medium to high antigenicity shows regression and the concentration of IL2 stabilizes. Unlike in (Kirschner and Panetta, 1998), the immune system also stabilizes, indicating that side effects like the capillary leak syndrome do not arise here. In other words, a patient does not need to endure very many side effects before the IL-2 therapy successfully clears the tumor. In (Rosenberg et al., 1994), the effectiveness of the high dose bolus treatment with interleukin-2 is studied, where many patients are in complete remission for 7 to 91 months. Hence, this model predicts that it is indeed possible to treat a patient cancer free with immunotherapy with IL-2 alone.

Finally, it can be said that the above finding sheds some light on immunotherapy with IL-2 and can be helpful to medical practitioners, experimental scientists and others to control this killer disease of cancer. An extension along this line of work will be to examine the effect of other cytokines such as IL-10, IL-12, interferon $-\gamma$, which are involved in the cellular dynamics of the immune system response to tumor invasion and how these cytokines affect the dynamics of the system.

\section{References}

Adam J. and N. Bellomo E. (1997). A Survey Models for TumorImmune System Dynamics, Birkhäuser, Boston, MA.

Banerjee S. and Sarkar R. R. (2008). Delay induced model for tumor-immune interaction and control of malignant tumor growth, Biosciences 91(1): 268-288.

Bodnar M. and Foryś U. (2000a). Behaviour of solutions to Marchuk's model depending on a time delay, International Journal of Applied Mathematics and Computer Science 10(1): 97-112.

Bodnar M. and Foryś U. (2000b). Periodic dynamics in the model of immune system, International Journal of Applied Mathematics and Computer Science 10(1): 113-126.

Bodnar M. and Foryś U. (2003a). Time delays in proliferation process for solid avascular tumor, Mathematical and Computer Modeling 37(11): 1201-1209.

Bodnar M. and Foryś U. (2003b). Time delays in regulatory apoptosis for solid avascular tumor, Mathematical and Computer Modeling 37(11): 1211-1220.

Byrne H. M. (1997). The effect of time delays on the dynamics of avascular tumor growth, Mathematical Biosciences 144(2): 83-117. 
Curti B. D., Ochoa A. C., Urba W. J., Alvord W. G., Kopp W. C., Powers G., Hawk C., Creekmore S. P., Gause B. L., Janik J. E., Holmlund J. T., Kremers P., Fenton R. G., Miller L., Sznol M., II J. W. S., Sharfman W. H. and Longo D. L. (1996). Influence of interleukin-2 regimens on circulating populations of lymphocytes after adoptive transfer of anti$c d 3$-stimulated $t$ cells: Results from a phase $i$ trial in cancer patients, Journal of Immunotherapy 19(4): 296-308.

Foryś U. (2002). Marchuk's model of immune system dynamics with application to tumor growth, Journal of Theoretical Medicine 4(1): 85-93.

Freedman H. I., Erbe L. and Rao V. S. H. (1986). Three species food chain models with mutual interference and time delays, Mathematical Biosciences 80(1): 57-80.

Freedman H. I. and Rao V. S. H. (1983). The trade-off between mutual interference and time lags in predator-prey systems, Bulletin of Mathematical Biology 45(6): 991-1004.

Galach M. (2003). Dynamics of the tumor-immune system competition-the effect of time delay, International Journal of Applied Mathematics and Computer Science 13(3): 395406.

Gause B. L., Sznol M., Kopp W. C., Janik J. E., II J. W. S., Steis R. G., Urba W. J., Sharfman W., Fenton R. G., Creekmore S. P., Holmlund J., Conlon K. C., VanderMolen L. A. and Longo, D. L. (1996). Phase $i$ study of subcutaneously administered interleuking-2 in combination with interferon alfa-2a in patients with advanced cancer, Journal of Clinical Oncology 14(8): 2234-2241.

Hale J. and Lunel S. (1993). Introduction to Functional Differential Equations, Springer-Verlag, New York, NY.

Hara I., Hotta H., Sato N., Eto H., Arakawa S. and Kamidono S. (1996). Rejection of mouse renal cell carcinoma elicited by local secretion of interleukin-2, Japanese Journal of Cancer Research 87(7): 724-729.

Kaempfer R., Gerez L., Farbstein H., Madar L., Hirschman O., Nussinovich R. and Shapiro A. (1996). Prediction of response to treatment in superficial bladder carcinoma through pattern of interleukin-2 gene expression, Journal of Clinical Oncology 14(6): 1778-1786.

Keilholz U., Scheibenbogen C., Stoelben E., Saeger H. D. and Hunstein W. (1994). Immunotherapy of metastatic melanoma with interferon-alpha and interleukin-2: Pattern of progression in responders and patients with stable disease with or without resection of residual lesions, European Journal of Cancer 30A(7): 955-958.

Kirschner D. and Panetta J. C. (1998). Modeling immunotherapy of the tumor - immune interaction, Journal of Mathematical Biology 37(3): 235-252.

Kolev M. (2003). Mathematical modeling of the competition between acquired immunity and cancer, International Journal of Applied Mathematics and Computer Science 13(3): 289-296.

Kuznetsov V. A., Makalkin I. A., Taylor M. A. and Perelson A. S. (1994). Nonlinear dynamics of immunogenic tumors: parameter estimation and global bifurcation analysis, Bulletin of Mathematical Biology 56(2): 295-321.
Matzavinos A., A.J.Chaplain M. and Kuznetsov V. A. (2004). Mathematical modelling of the spatio-temporal response of cytotoxic $t$-lymphocytes to a solid tumor, Mathematical Medicine and Biology 21(1): 1-34.

Rabinowich H., Banks M., Reichert T. E., Logan T. F., Kirkwood J. M. and Whiteside T. L. (1996). Expression and activity of signaling molecules in $t$-lymphocytes obtained from patients with metastatic melanoma before and after interleukin-2 therapy, Clinical Cancer Research 2(8): 1263-1274.

Rosenberg S. A. and Lotze M. T. (1986). Cancer immunotherapy using interleukin-2 and interleukin-2-activated lymphocytes, Annual Review of Immunology 4(1): 681-709.

Rosenberg S. A., Yang J. C., Topalian S. L., Schwartzentruber D. J., Weber J. S., Parkinson D. R., Seipp C. A., Einhorn J. H. and White D. E. (1994). Treatment of 283 consecutive patients with metastatic melanoma or renal cell cancer using high-dose bolus interleukin 2, Journal of the American Medical Association 271(12): 907-913.

Rosenstein M., Ettinghousen S. E. and Rosenberg S. A. (1986). Extravasion of intravascular fluid mediated by the systemic administration of recombinant interleukin-2, Journal of Immunology 137(5): 1735-1742.

Sarkar R. R. and Banerjee S. (2005). Cancer self remission and tumor stability — A stochastic approach, Mathematical Biosciences 196(1): 65-81.

Schwartzentruber D. J. (1993). In vitro predictors of clinical response in patients receiving interleukin-2-based immunotherapy, Current Opinion in Oncology 5(6): 1055-1058.

Szymańska Z. (2003). Analysis of immunotherapy models in the context of cancer dynamics, International Journal of Applied Mathematics and Computer Science 13(3): 407418.

Tartour E., Blay J. Y., Dorval T., Escudier B., Mosseri V., Douillard J. Y., Deneux L., Gorin I., Negrier S., Mathio C., Pouillart P. and Fridman W. H. (1996). Predictors of clinical response to interleukin-2-based immunotherapy in melanoma patients: A French multi-institutional study, Journal of Clinical Oncology 14(5): 1697-1703.

Yang X., Chen L. and Chen J. (1996). Permanence and positive periodic solution for the single-species nonautonomous delay diffusive model, Computers and Mathematics with Applications 32(4): 109-116.

Zhivkovc P. and Waniewski J. (2003). Modeling tumorimmunity interactions with different stimulation functions, International Journal of Applied Mathematics and Computer Science 13(3): 307-315.

\section{Appendix}

Nyquist Criterion If $L$ is the arc length of a curve encircling the right half plane, the curve $\bar{p}_{J}(L)$ will encircle the origin as many times number as the difference between the number of poles and the number of zeroes of $\bar{p}_{J}(L)$ in the right half-plane.

Received: 3 August 2007

Revised: 22 February 2008 